\title{
In vitro assessment of a calcium-fluoroaluminosilicate glass-based desensitizer for the prevention of root surface demineralization
}

\author{
Hiroyuki MIYAJIMA¹, Takuya ISHIMOTO², Sai MA², Jihua CHEN³, Takayoshi NAKANO² and Satoshi IMAZATO'1 \\ ${ }^{1}$ Department of Biomaterials Science, Osaka University Graduate School of Dentistry, Suita, 1-8 Yamadaoka, Suita, Osaka 565-0871, Japan \\ ${ }^{2}$ Department of Materials Science and Engineering, Osaka University Graduate School of Engineering, Suita, 1-8 Yamadaoka, Suita, Osaka 565- \\ 0871, Japan \\ ${ }^{3}$ Department of Prosthodontics, School of Stomatology, Fourth Military Medical University, Xi'an, 710032, P.R. China \\ Corresponding author, Satoshi IMAZATO; E-mail: imazato@dent.osaka-u.ac.jp
}

\begin{abstract}
The purpose of this study was to evaluate the ability of a calcium-fluoroaluminosilicate glass-based desensitizer (Nanoseal) to protect against root demineralization in vitro. Nanoseal was applied to human root dentin, which was immersed in acidic buffer for $72 \mathrm{~h}$, or exposed to $\mathrm{pH}$ cycling by immersing in distilled water or mineralizing solution for $24 \mathrm{~h}$ intermediately during $48 \mathrm{~h}$-acid attack. Demineralization was evaluated by $\mu$-CT, and mineral loss (ML) and lesion depth (LD) were determined from mineral density profiles. ML and LD in all treatment groups were significantly smaller compared with control. The Nanoseal-treated group with $\mathrm{pH}$ cycling using mineralizing solution had the lowest ML and LD. Analysis using an EPMA demonstrated calcium and phosphorous were incorporated into the superficial layer of specimens in the Nanoseal-treated groups, suggesting Nanoseal modified the dentin surface, making it resistant to demineralization. Application of Nanoseal is an effective method for protecting root from demineralization.
\end{abstract}

Keywords: Root surface, Demineralization, Desensitizer, Micro-computed tomography

\section{INTRODUCTION}

The elderly can now retain their teeth until much later in life owing to the increased awareness of oral hygiene, use of dental care, or improved preventive strategies ${ }^{1}$. However, the prevalence of root caries is increasing as the population ages $^{2-4)}$. Gingival recession, which makes the retained dentition susceptible to root surface caries, is caused by normal aging, periodontal disease or traumatic toothbrushing habits ${ }^{5,6}$. Additionally, the risk of developing root surface caries is further increased by reduced salivary flow or xerostomia caused by aging or certain medications ${ }^{7}$.

Restoration of root surface caries can be problematic because of its proximity to the gingiva, where isolation and access for the placement of restorative materials are difficult. Additionally, the treatment time should be shortened and local anesthesia cannot be used in many cases because of physical problems, especially for institutionalized elderly. Considering that lesion progression in roots is faster than in enamel because of their relatively low mineral content ${ }^{8,9)}$, it is therefore recommended that caries preventive measures should be taken immediately after exposure of root surfaces.

Fluoride is widely accepted as an effective caries preventative agent ${ }^{10)}$, owing to the inhibition of the physicochemical ${ }^{11)}$ and microbiological ${ }^{12)}$ processes of root caries initiation and development. Fluoride-containing mouthrinses, toothpastes and topical varnishes are recommended by clinicians as a prophylactic program to high-risk populations ${ }^{13-16)}$. However, the effects of these

Color figures can be viewed in the online issue, which is available at J-STAGE.

Received Aug 19, 2015: Accepted Dec 22, 2015

doi:10.4012/dmj.2015-273 JOI JST.JSTAGE/dmj/2015-273 preventive strategies are temporary and successful prevention of root surface caries is hindered by the low compliance of patients. Thus, there is a great need for new methods that can inhibit the development of root surface caries for long-term periods, with a simple application.

The dentin desensitizer Nanoseal is composed of calcium-fluoroaluminosilicate glass and phosphoric acid, which set upon mixing of the components, in a conventional acid-base reaction. The aggregates of phosphoric acid and silicate glass precipitate on the dentin surface immediately after application. Such acidic aggregates demineralize the dentin surface and incorporate various ions, such as calcium or phosphate, derived from the tooth component. Subsequently, a nanoparticle-deposited layer, of approximately 1-2 $\mu \mathrm{m}$ thickness, containing phosphoric silicate, calcium fluoride, or calcium phosphate, is formed on the dentin surface.

Previous studies reported that enamel and dentin surfaces treated with Nanoseal exhibited a resistance to acid demineralization ${ }^{17,18}$. Considering the formation process of the nanoparticle-deposited layer by Nanoseal, incorporating fluorine and silica, it is presumed that this material actively interacts with dentin. This interaction results in modification of the dentinal surface to make it less sensitive to acid, rather than providing a physical seal. Although Nanoseal has been marketed to treat dentinal hypersensitivity, it is hypothesized that this material could protect root surface caries, owing to its ability to modify the dentinal surface. However, few studies have examined the preventive effects of Nanoseal against root surface demineralization. Additionally, considering its 
possible capacity to enable ion permeability, Nanoseal might also exhibit remineralizing effects.

The purpose of this study was to evaluate the ability of the calcium-fluoroaluminosilicate glassbased desensitizer Nanoseal to protect the root dentin from demineralization in vitro using micro-computed tomography (micro-CT).

\section{MATERIALS AND METHODS}

\section{Specimen preparation}

Twenty-four human extracted molars with intact roots were used under the approval of the Human Research Ethics Committee Osaka University Graduate School of Dentistry, Japan (approval number: H25-E23). The cementum was removed using a periodontal scaler to expose root dentin, and the root was separated from the crown, 1-2 $\mathrm{mm}$ below the cemento-enamel junction, using a low-speed diamond saw (Isomet 2000, Buehler, Lake Bluff, IL, USA) under water coolant. Cubic root specimens (approximately $5 \mathrm{~mm}^{3}$ ) were prepared and coated with four layers of an acid-resistant nail varnish, leaving a window of $3 \mathrm{~mm}^{2}$ exposed dentin (Fig. 1a). Buccal area of the tooth was selected for making the window. The specimens were randomly divided into two groups (12 teeth each). For the experimental group, Nanoseal (Nippon Shika Yakuhin, Yamaguchi, Japan) was applied according to the manufacturer's instructions. Briefly, two liquids were thoroughly mixed using a micro-brush, applied to the dentin surface for 20 $\mathrm{s}$, and washed with water. The specimens in the control group were left uncoated.

\section{Demineralization}

Specimens in the Nanoseal-applied or control groups were immersed individually in $10 \mathrm{~mL}$ acidic demineralizing buffer solution at $\mathrm{pH} 4.5$, and stored at $37^{\circ} \mathrm{C}$ for 3 days. The solution contained $2.2 \mathrm{mmol} / \mathrm{L} \mathrm{CaCl}_{2}, 2.2 \mathrm{mmol} / \mathrm{L}$
$\mathrm{NaH}_{2} \mathrm{PO}_{4}, 50 \mathrm{mmol} / \mathrm{L}$ acetic acid, and $\mathrm{NaOH}$ to adjust $\mathrm{pH}^{19)}$. The demineralization buffer was changed every 24 h. Four specimens were tested for each group.

Demineralization tests were also conducted using a $\mathrm{pH}$ cycle protocol. The Nanoseal-applied or control specimens were immersed in $10 \mathrm{~mL}$ acidic demineralizing solution at $37^{\circ} \mathrm{C}$ as described above. After $24 \mathrm{~h}$, they were transferred into $10 \mathrm{~mL}$ of distilled water $(\mathrm{pH}$ 6.3) or mineralizing solution at $\mathrm{pH} 7.0(0.02 \mathrm{~mol} / \mathrm{L} \mathrm{HEPES}$, $3.0 \mathrm{mmol} / \mathrm{L} \mathrm{CaCl}$ and $\left.1.8 \mathrm{mmol} / \mathrm{L} \mathrm{KH}_{2} \mathrm{PO}_{4}\right)^{20)}$, and stored at $37^{\circ} \mathrm{C}$ for $24 \mathrm{~h}$. Then, the specimens were placed again in $10 \mathrm{~mL}$ acidic demineralizing solution for $24 \mathrm{~h}$ at $37^{\circ} \mathrm{C}$. Four specimens were tested for each group.

\section{Micro-CT analysis}

Demineralization was evaluated using a micro-focused X-ray CT (SMX-100CT-SV3, Shimadzu, Kyoto, Japan), which can generate polychromatic X-rays with a conebeam geometry. The specimen was mounted on a computer-controlled turntable with the treated dentin surface parallel to the X-ray beam, and was covered with a wet cotton roll to prevent desiccation. The scanning was performed with the specimen being rotated $360^{\circ}$ in steps of $0.6^{\circ}$. The tube voltage was $90 \mathrm{kV}$, at a current of $29 \mathrm{~mA}$. The distance from the X-ray source to the sample and detector was 78.5 and $494.9 \mathrm{~mm}$, respectively.

Using a commercial volume graphics software (VGStudio, Volume Graphics, Heidelberg, Germany), cross-sectional images of the two perpendicular planes within the area where the material had been applied were obtained and the results were compared in $2 \mathrm{D}$ level.

Then, the three-dimensional (3D) image of each scanned specimen was reconstructed using the same software. For the purpose of mineral density calibration, mineral reference phantom (Phantom, Ratoc System Engineering, Tokyo, Japan) was scanned prior to specimen scanning. Phantom contained hydroxyapatite (a)

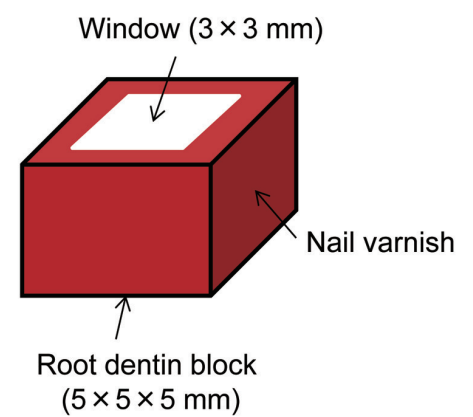

(b)

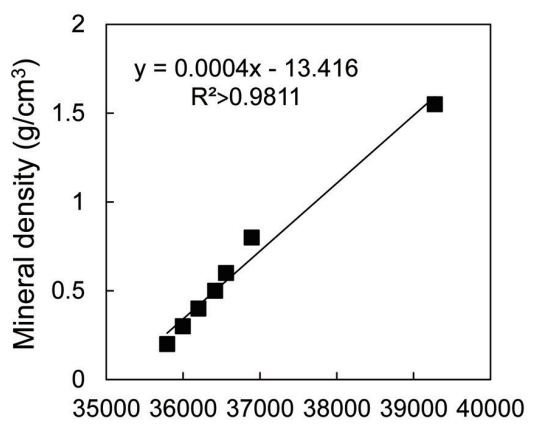

(c)

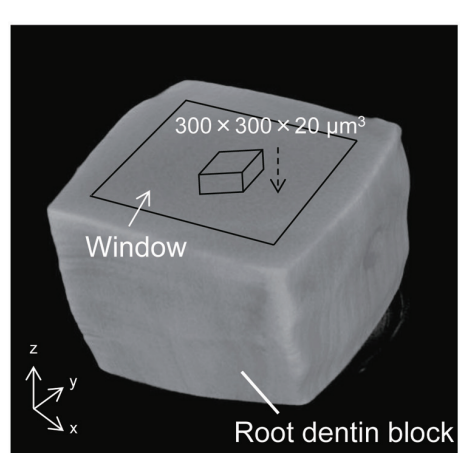

Fig. 1 (a) Schematic illustration of a cubic root specimen coated with an acid-resistant nail varnish, leaving a window for demineralization. (b) Mineral density calibration curve based on 16-bit gray values obtained from the mineral reference phantom (linear regression, $R^{2}>0.9811$ ). (c) Three-dimensional computed tomography image of a root dentin block.

Each density label for the measurement of mineral density was set through the dentin surface in a volume of interest $(\mathrm{VOI})$ of $300 \times 300 \times 20 \mu \mathrm{m}$, in the center of demineralization window. 
disks $\left(0.2,0.3,0.4,0.5,0.6,0.8\right.$ and $\left.1.55 \mathrm{~g} / \mathrm{cm}^{3}\right)$, which was scanned using the same setup and parameters as for the tooth specimens. The data in each scan were acquired as 600 images in 16-bit TIFF format, and used to reconstruct a $3 \mathrm{D}$ image stack from the $2 \mathrm{D}$ images, with a resolution of $512 \times 512$ pixels and an isotropic volumetric pixel (voxel) size of $20 \mu \mathrm{m}$, using 3D analysis software (TRI/3D-BON, Ratoc System Engineering, USA). CT values were converted into mineral density values $\left(\mathrm{g} / \mathrm{cm}^{3}\right)$ using a linear calibration curve based on the gray values obtained from the mineral reference phantom (linear regression, $\mathrm{R}^{2}>0.9811$ ) as illustrated in Fig. 1b. A noise reducing median filter was applied to the data, and the background was removed by excluding those pixels with a CT value lower than the zero mineral density value according to the calibration curve (Fig. 1b). The rendered 3D volumes were manually translated and rotated in the software to visually match the baseline image. The features on each image used for the matching process were the sound dentin surface from specimen edges. The assessment was performed in a volume of interest (VOI) of $300 \times 300 \times 20 \mu \mathrm{m}$, at the center of demineralization window (Fig. 1c). Mineral density values (MD) were calculated in the VOI, at 20 $\mu \mathrm{m}$ depth. MD profiles for each specimen were obtained by plotting the MD against the depth. For each specimen of the experimental group, micro-CT scanning was performed before/after application of Nanoseal and after demineralization. Lesion depth (LD; $\mu \mathrm{m})$ after $72 \mathrm{~h}$ was calculated from the MD profiles. $\mathrm{LD}$ was recorded as the depth of the region where the mineral content reached $95 \%$ of the maximum mineral density ${ }^{21)}$. Mineral loss $\left(\mathrm{ML} ; \mathrm{mm}^{3}\right.$ ) after $72 \mathrm{~h}$ was calculated using 3D analysis software (TRI/3D-BON).

\section{Scanning electron microscope (SEM) observation}

The Nanoseal was applied to the exposed dentin surface of the root as described above. The specimens were rinsed with PBS and fixed in 2.5\% glutaraldehyde. After dehydration in ascending grades of ethanol (50, 70, 80, and $100 \%$ ), they were gold-coated using an ion sputtering device (SC-701 QUICK COATER, Sanyu Electron, Tokyo, Japan) and examined with scanning electron microscopy (JSM-6390 BU, JEOL, Tokyo, Japan; SEM) at an accelerating voltage of $15 \mathrm{kV}$.

\section{Electron probe micro analyzer (EPMA) examination}

Specimens were dehydrated through a graded series of ethanol solutions, deposited in propylene oxide, and embedded in acrylic resin (Ostron, GC, Tokyo, Japan). The resin blocks containing the specimens were cut sagittally using a low speed diamond micro-cutter (Isomet, Buehler). All specimens were polished with silicone carbide paper (\#1500-grit, Maruto, Tokyo, Japan), with a water coolant. The specimens were dried in vacuum desiccator, sputtered with white gold using an auto fine coater (JFC-1600, JEOL), and examined with Electron Probe X-ray Micro Analyzer (EPMA$1720 \mathrm{H}$, Shimadzu).

The electron microprobe settings were $15 \mathrm{kV}$ (accelerating voltage) and $100 \mathrm{nA}$ (probe current). A scanning spot with a spatial resolution of $640 \times 480$ pixels and a dwell time of 2.2 milliseconds per point were used for the elemental mapping. The range of measurement was $832 \times 624 \mathrm{~mm}$. The standards used for calibration of the microanalysis system were calcium and phosphorus.

\section{Statistical analysis}

Statistical analysis of LD and ML results was accomplished by one-factor analysis of variance (ANOVA) with a Scheffe test. For all analyses, values of $p<0.05$ were considered statistically significant.

\section{RESULTS}

\section{Micro-CT analysis}

Figure 2 shows typical 3D images of the control and experimental specimens, before and after immersion in acidic buffer ( $\mathrm{pH} 4.5$ ) for $72 \mathrm{~h}$. Nanoseal treatment did not produce any visible layer (Fig. 2c). After demineralization for $72 \mathrm{~h}$, a radiolucent concave-shaped superficial lesion was observed in the specimen of the control group (Fig. $2 b)$. In contrast, slight demineralization was observed for the Nanoseal-treated specimen (Fig. 2d). The same results were obtained for all four specimens tested. Similar to continuous acid attack for $72 \mathrm{~h}$, slight demineralization was observed for the Nanoseal-treated specimen, after demineralization by $\mathrm{pH}$ cycling using distilled water or mineralizing solution, compared with the control (data not shown).

Figure 3 shows cross-sectional images of the specimens displayed in Fig. 2. After demineralization for $72 \mathrm{~h}$, a radiolucent decalcification lesion was observed in the control group (Fig. 3a). In contrast, a superficial zone, with some increased resistance against demineralization, was observed in the Nanoseal-treated group (Fig. 3b). Similar to the result of the $72 \mathrm{~h}$-continuous acid attack, slight demineralization was observed for the Nanosealtreated specimen after demineralization by $\mathrm{pH}$ cycling, using distilled water or mineralizing solution, compared with the control (data not shown).

\section{Mineral density profiles and lesion depth}

The representative MD profiles for each group are indicated in Fig. 4. After continuous acid attack for $72 \mathrm{~h}$, the MD profile of the Nanoseal-treated specimen showed some resistance against demineralization compared with the control specimen, and a subsurface layer with increased resistance was detected in the Nanosealtreated specimen (Fig. 4a). After demineralization by $\mathrm{pH}$ cycling using distilled water, in which the total acidimmersion time was decreased to $48 \mathrm{~h}$, the MD profiles of the Nanoseal-treated specimen showed resistance against demineralization (Fig. 4b). The resistance against demineralization was also clearly observed in the MD profiles of the Nanoseal-treated specimen after demineralization by $\mathrm{pH}$ cycling using mineralizing solution (Fig. 4c).

The LD values of each group are shown in Fig. 

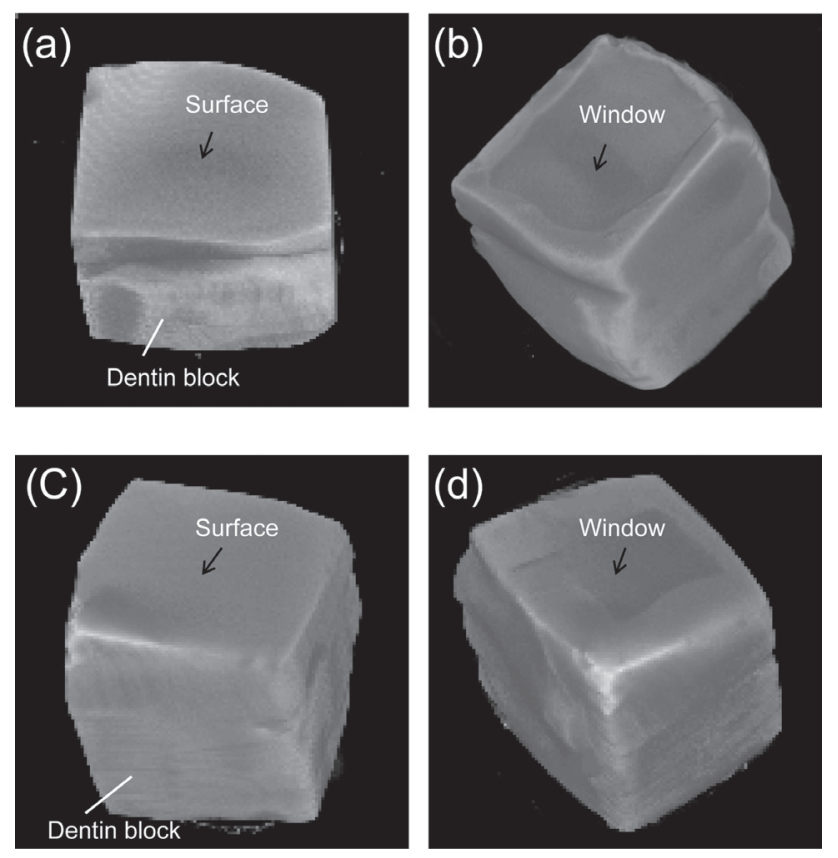

Fig. 2 Typical 3D images of the specimen immersed in acidic buffer at $\mathrm{pH} 4.5$ for $72 \mathrm{~h}$.

(a): control before demineralization (b): control after demineralization (c): Nanoseal-treated specimen before demineralization (d): Nanoseal-treated specimen after demineralization. After demineralization for $72 \mathrm{~h}$, a radiolucent concave shaped superficial lesion was observed in the specimen of the control group (b). Slight demineralization was observed for the Nanoseal-treated specimen (d).

(a)
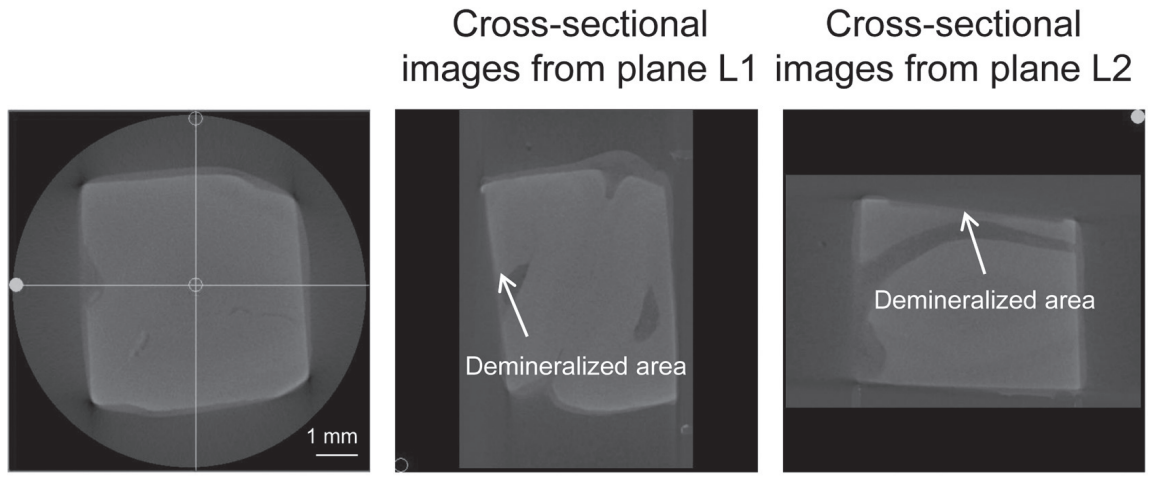

Cross-sectional images from plane L1 images from plane L2

(b)
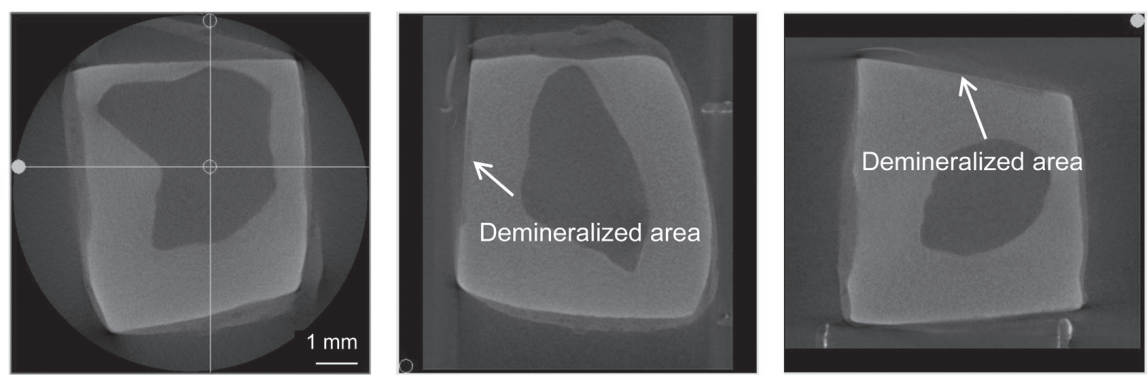

Fig. 3 Typical cross-sectional images of the specimens immersed in acid buffer at $\mathrm{pH} 4.5$ for $72 \mathrm{~h}$. The two lines, L1 and L2, represent the two perpendicular planes from which the crosssectional images were obtained. (a): control (b): Nanoseal-treated specimen. After demineralization for $72 \mathrm{~h}$, a radiolucent decalcification lesion was observed in the specimen of the control group (a). In contrast, a superficial zone with some increased resistance against demineralization could be observed in the Nanoseal-treated group (b). 
(a)

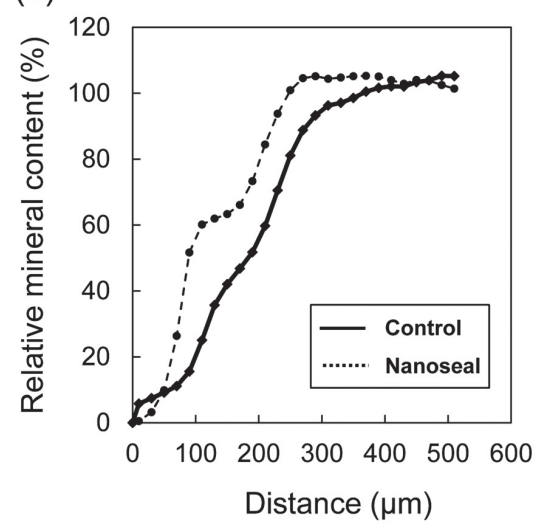

(b)

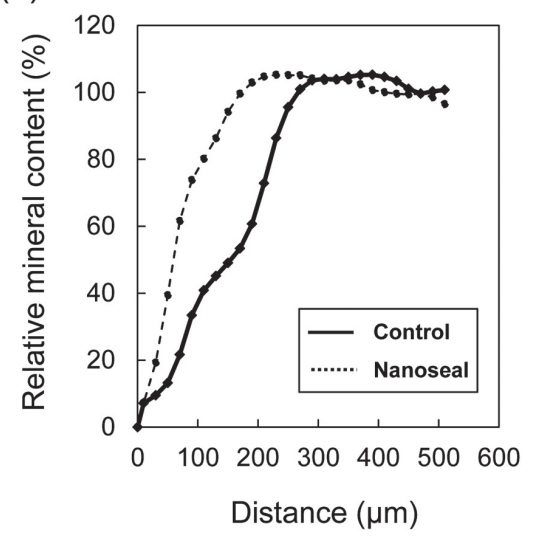

(c)

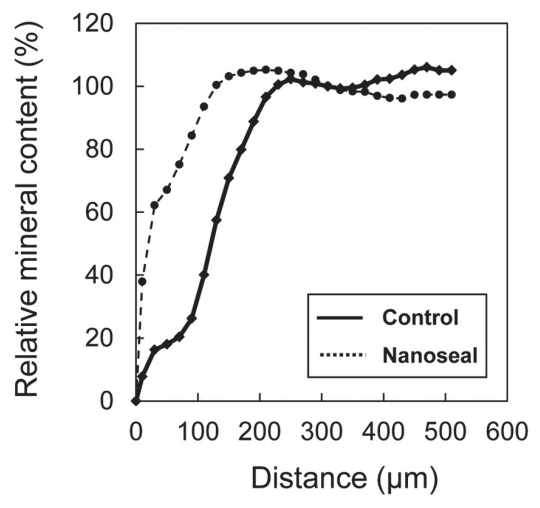

Fig. 4 Mineral density profiles for each group.

(a) control and Nanoseal-treated specimens after $72 \mathrm{~h}$ of demineralization, (b) control and Nanoseal-treated specimens after demineralization with $\mathrm{pH}$ cycling using distilled water, (c) control and Nanoseal-treated specimen after demineralization with $\mathrm{pH}$ cycling using mineralizing solution.

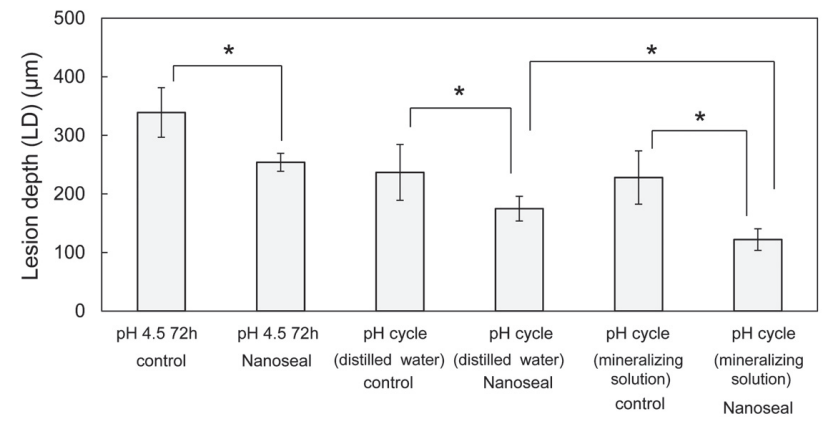

Fig. 5 Lesion depth (LD; $\mu \mathrm{m})$ for each group.

Results are expressed as mean \pm standard deviation (SD). Control shows the specimens without application of Nanoseal.

5. Under all conditions with/without $\mathrm{pH}$ cycling, LD values for the Nanoseal-treated group were significantly smaller compared with the control $(p<0.05)$. In addition, significantly smaller LD values were observed for the Nanoseal-treated group with $\mathrm{pH}$ cycling using mineralizing solution compared with distilled water $(p<0.05)$.

\section{Mineral loss}

The ML values for the groups with the application of Nanoseal were significantly smaller than those in the control group, under all demineralization protocols ( $p<0.05$, Fig. 6). The Nanoseal-treated group treated with $\mathrm{pH}$ cycling using mineralizing solution demonstrated significantly lower ML values compared with those using distilled water $(p<0.05)$.

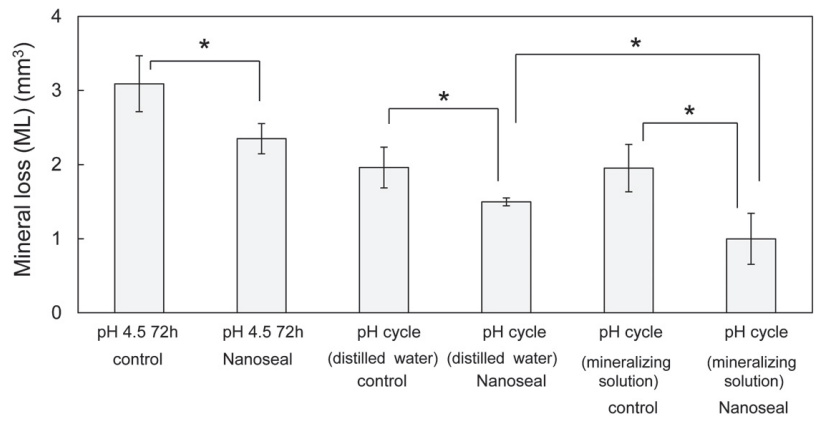

Fig. 6 Mineral loss (ML) $\left(\mathrm{mm}^{3}\right)$ for each group. Results are expressed as means and standard deviations (SD). Control shows the specimens without application of Nanoseal.

\section{SEM observation}

SEM images of the specimens with or without Nanoseal treatment are presented in Fig. 7. Formation of deposits on the dentin surface by the treatment with Nanoseal can be clearly seen (Fig. 7b).

\section{EPMA examination}

The EPMA images obtained for calcium and phosphorus are shown in Figs. 8 and 9. After $72 \mathrm{~h}$ of demineralization, the incorporation of calcium and phosphorus in the superficial layer of the lesion (white arrow) was detected to a small extent in the Nanoseal-treated specimens, though neither calcium nor phosphorus were observed in the control group (Figs. 8a,b; 9a,b). In the case of the specimens treated with $\mathrm{pH}$ cycling using distilled water, the Nanoseal-treated specimen demonstrated spotted deposits of calcium and phosphorus on the surface of the lesion (Figs. 8d, 9d), while such deposits were not 
(a)

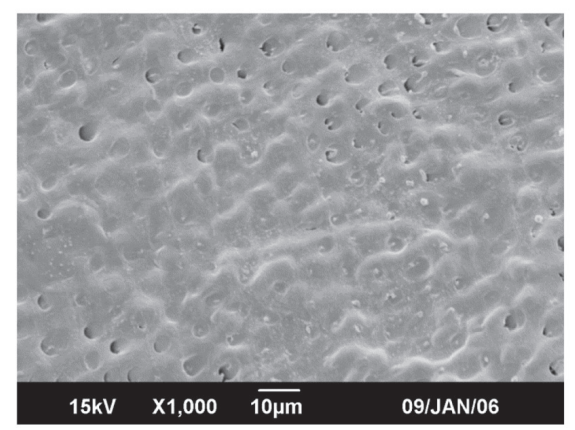

(b)

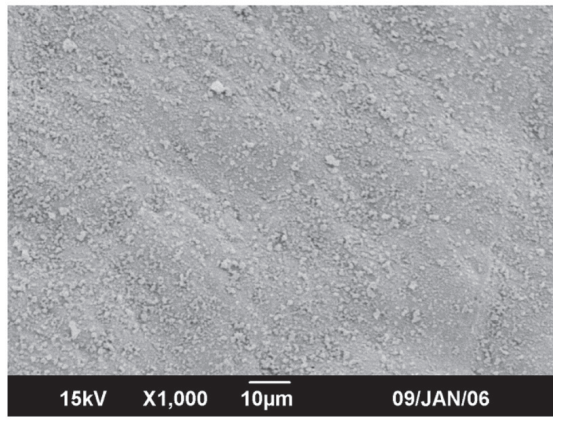

Fig. 7 SEM images of the exposed dentin surface of the root $(\times 1,000)$ without (a) or with (b) Nanoseal-treatment. Scale bar $=10 \mu \mathrm{m}$.

(a)

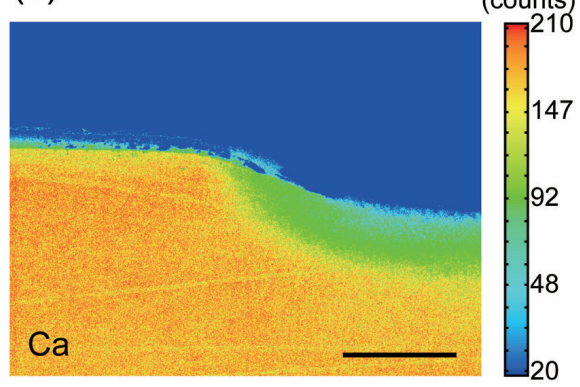

(c)

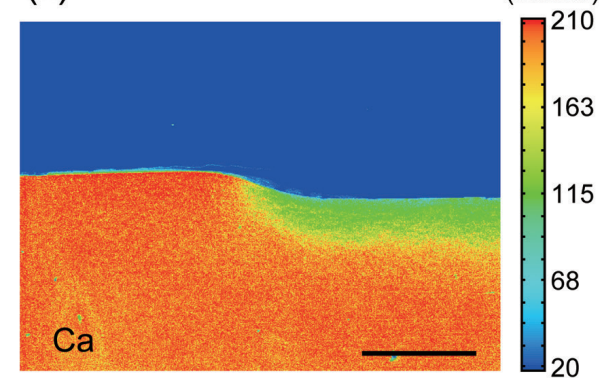

(e)

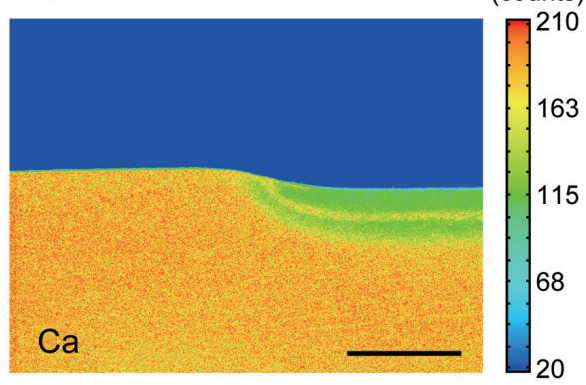

(b)

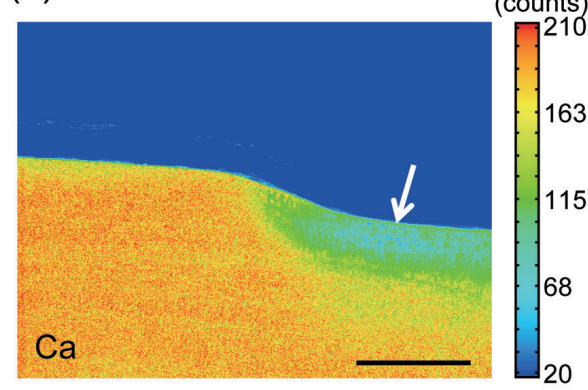

(d)

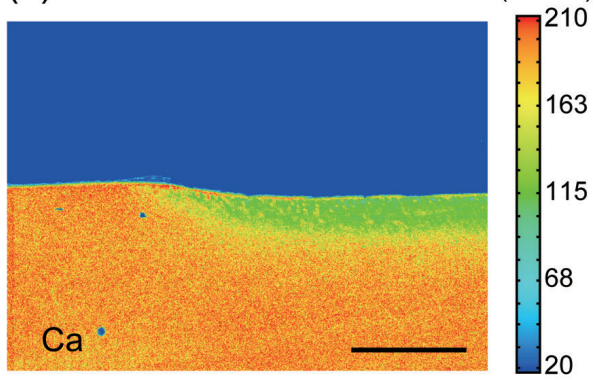

(f)

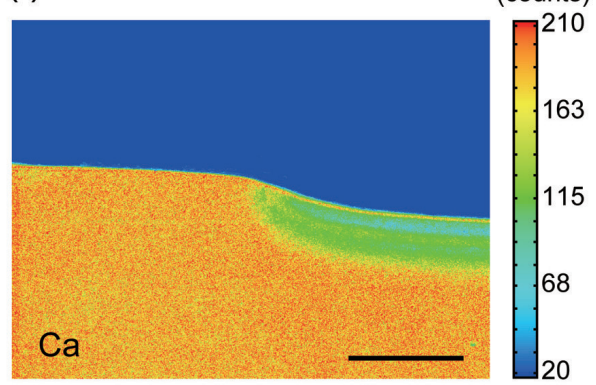

Fig. 8 Electron probe micro-analyzer images for calcium for each group.

(a) control after $72 \mathrm{~h}$ of demineralization, (b) Nanoseal-treated specimen after 72 $\mathrm{h}$ of demineralization, (c) control after demineralization with $\mathrm{pH}$ cycling using distilled water, (d) Nanoseal-treated specimen after demineralization with $\mathrm{pH}$ cycling using distilled water, (e) control after demineralization with $\mathrm{pH}$ cycling using mineralizing solution, (f) Nanoseal-treated specimen after demineralization with $\mathrm{pH}$ cycling using mineralizing solution. Scale bar $=200 \mu \mathrm{m}$. 

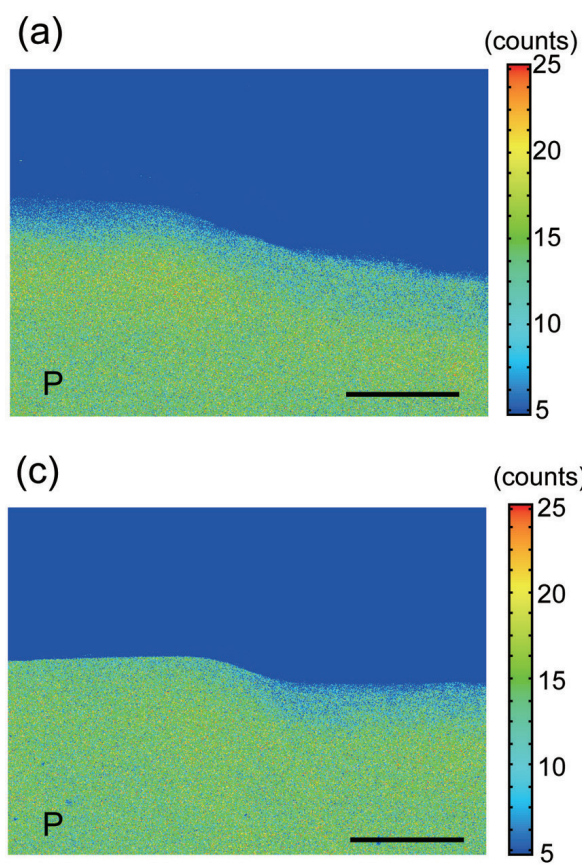

(e)

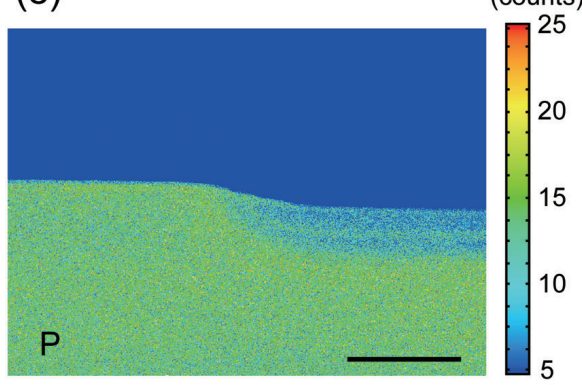

(b)

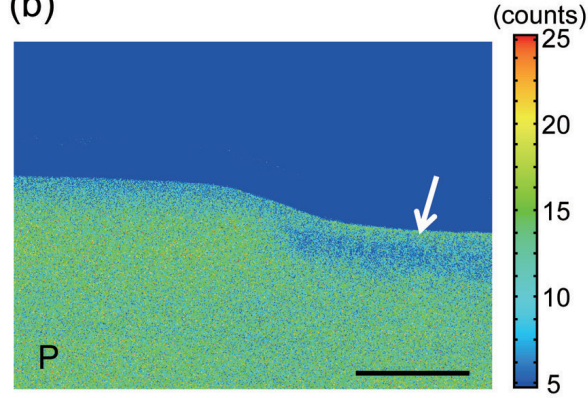

(d)
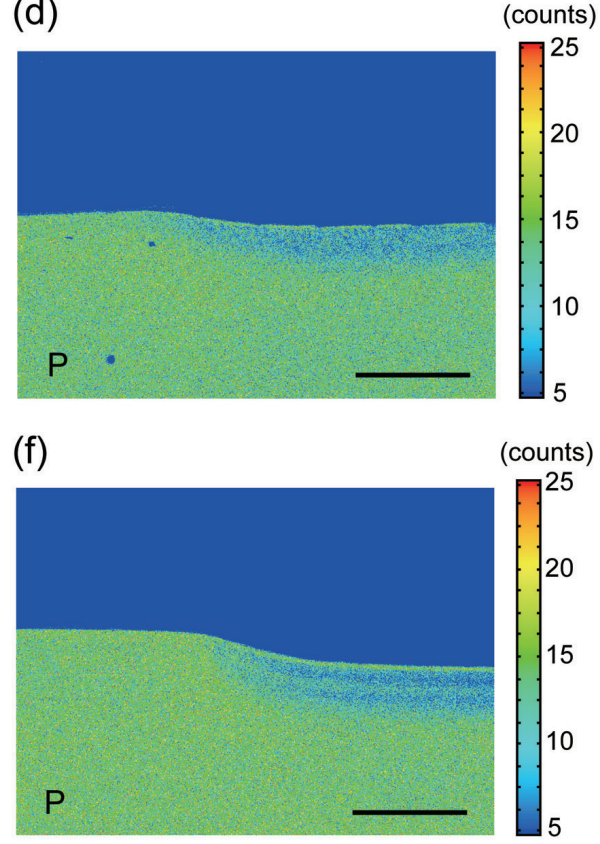

Fig. 9 Electron probe micro-analyzer images for phosphorous for each group.

(a) control after $72 \mathrm{~h}$ of demineralization in acidic buffer, (b) Nanoseal-treated specimen after $72 \mathrm{~h}$ of demineralization, (c) control after demineralization with $\mathrm{pH}$ cycling using distilled water, (d) Nanoseal-treated specimen after demineralization with $\mathrm{pH}$ cycling using distilled water, (e) control after demineralization with $\mathrm{pH}$ cycling using mineralizing solution, (f) Nanoseal-treated specimen after demineralization with $\mathrm{pH}$ cycling using mineralizing solution. Scale bar=200 $\mu \mathrm{m}$.

observed for the control (Figs. 8c, 9c). Under pH cycling using mineralizing solution, deposition of calcium and phosphorus to some extent in the lesion was found for the control group (Figs. 8e, 9e). However, a calcium- and phosphorus-rich superficial layer was clearly observed only for the Nanoseal-treated specimen (Figs. 8f, 9f).

\section{DISCUSSION}

The exposure of root dentin after gingival recession is very common among the elderly population, because the thin cementum can be easily removed by intensive root planing or over-zealous toothbrushing ${ }^{20)}$. Therefore, root specimens without cementum were used in the present study. Demineralization of the tooth surface has been investigated through various methods, including contact microradiography ${ }^{22)}$, polarized light microscopy ${ }^{23)}$, nanoindentation testing ${ }^{24}$, confocal laser microscopy ${ }^{25)}$, and micro- $\mathrm{CT}^{26)}$. Among these, contact microradiography has been accepted as the standard technique for determination of mineral content and caries lesion depth. However, specimen preparation for all these methods is destructive and the results are dependent upon the information obtained from thin sections. In contrast, micro-CT can non-destructively create $3 \mathrm{D}$ images from a bulk specimen, also providing cross-sectional information. Furthermore, artifacts resulting from the preparation of thin sections are removed from the process, and the 3D reconstruction enables the evaluation of any desired location throughout the scanned specimen ${ }^{27}$. This non-destructive micro-CT technique is useful for the evaluation of mineral content of the same specimen 
at different time points. Moreover, by taking advantage of mineral reference phantoms, precise information of mineral density in teeth can be readily quantified ${ }^{28}$. A recent report concluded that micro-CT has the potential to substitute transverse microradiography (TMR) for in vitro studies on dental caries ${ }^{29)}$. A mineral reference phantom was used to determine mineral density in this study. The intense linear regression of the mineral density calibration curve, and its reproducibility, demonstrated the adequacy of the mineral determination technique and the experimental set-up, compared with the previous studies ${ }^{30,31)}$.

Nanoseal, the desensitizer examined in this study, is composed of calcium-fluoroaluminosilicate glass and phosphoric acid. It has a similar composition to silicate cements, which are predecessors of glass-ionomer cements. The setting reaction of Nanoseal involves an acid-base reaction that is initiated upon mixing of the components. During mixing and its application to dentin, two reactions proceed; one is the reaction between phosphoric acid and the glass components, and the other occurs between Nanoseal and dentin. Phosphoric acid dissolves calcium-fluoroaluminosilicate glass, while aluminum phosphate, calcium phosphate, calcium fluoride and silicate compounds are subsequently formed in the intergranular space. These phosphates and fluorides are combined with silicate compounds, which ultimately form a nucleated structure in the same way as silicate cement ${ }^{32}$. By this reaction, the $\mathrm{pH}$ of Nanoseal increases, while phosphate, fluoride or silicate compound particles are deposited on the tooth surface. Simultaneously, the tooth surface is etched by phosphoric acid from Nanoseal, and minerals are released from the tooth. Consequently, the $\mathrm{pH}$ on the tooth surface increased. Through these processes, an area of particle deposition with low solubility is finally formed.

In this study, when the specimens were immersed in acidic buffer continuously for $72 \mathrm{~h}$, significant inhibition of decalcification of root dentin was observed in the group with Nanoseal application. Nanoseal contains calcification-related elements, such as calcium and fluorine. Silica is also suggested to induce dentin remineralization $^{33)}$. Previous studies reported that these elements were absorbed onto the tooth surface by Nanoseal application ${ }^{17,18)}$. It is well known that fluoride application is effective for preventing dental caries, because it promotes production of fluoroapatite with improvement of the crystallinity of enamel ${ }^{34-36)}$. It is speculated that the transfer of ions, such as calcium, fluorine, phosphate or silica, from Nanoseal to the tooth may induce calcification and increased acid resistance ${ }^{17)}$. In fact, SEM results confirmed that mineral deposits are formed on the dentin surface by applying Nanoseal, which may be effective to resist against acid attack. To further investigate the protective effects of Nanoseal against demineralization, we analyzed the elemental distribution of the root dentin surface using EPMA. In the superficial layer of the Nanoseal-treated group specimen, slight residuals of calcium and phosphorus were detected after $72 \mathrm{~h}$-acid attack. This subsurface decalcification indicates that Nanoseal possesses a resistance to demineralization. By EPMA analysis, no clear difference in the lesion depth was seen between the control and Nanoseal-treated groups for 72 h-acid attack protocol. This is probably due to the differences in sample preparation. Micro-CT image was obtained without drying the specimens, but dehydration treatment was conducted for EPMA analysis. In addition, EPMA images only indicate the results of the middle part of the lesion.

In general, exposure of the root dentinal surface subjects it to a continuous demineralization and remineralization process. If demineralization dominates, it may lead to progression of a caries lesion. Considering the ion permeability of the Nanoseal layer, we then examined its ability to inhibit demineralization of the root surface with employment of a $\mathrm{pH}$ cycling protocol using mineralizing solution $(\mathrm{pH}$ 7.0) and compared the results with those obtained using distilled water $(\mathrm{pH}$ 6.3). For both specimens, with or without Nanoseal application, the $\mathrm{LD}$ and $\mathrm{ML}$ values were lower for the $\mathrm{pH}$ cycle using distilled water compared with the specimens exposed to $72 \mathrm{~h}$ of demineralization. This result confirmed the time-response effect of demineralization, as the total time of acid immersion was simply reduced by two thirds during the $\mathrm{pH}$ cycling using distilled water. EPMA analysis of the specimens with $\mathrm{pH}$ cycling using distilled water revealed partial existence of calcium and phosphorus in the superficial layer of the lesion for the Nanoseal-treated group, while complete loss of these ions occurred in the control group. This clearly indicates that an acid resistant layer was formed by Nanoseal application.

It is noteworthy that after demineralization by $\mathrm{pH}$ cycling using mineralizing solution, the Nanosealtreated group showed lower $\mathrm{LD}$ and $\mathrm{ML}$ values than those observed using distilled water. Deposits of calcium and phosphorous on the surface of the demineralized lesions in the Nanoseal-treated group were also clearly demonstrated by EPMA. These findings suggest that the Nanoseal layer on the tooth surface exhibits effective ion permeability for remineralization. Thus, these results indicate that the tooth surface is modified by Nanoseal application to resist against acid and to allow permeation of remineralizing components, consequently inhibiting decalcification of dentin.

To evaluate clinical effectiveness, further investigation using an in situ model, simulating oral conditions with natural saliva and dental plaque, is needed. Additionally, to establish preventive measures beneficial for elderly people, the durability of protective measures should be of high importance. Furthermore, the wear resistance of Nanoseal using the toothbrush abrasion test and the durability of its inhibitory effects on demineralization after brushing will be further investigated in future studies. 


\section{CONCLUSIONS}

A calcium-fluoroaluminosilicate glass-based desensitizer, Nanoseal, was effective in preventing demineralization of root dentin in vitro, and inhibited demineralization by acid to a greater extent when subjected to $\mathrm{pH}$ cycling using mineralizing solution compared with control specimens. This protection may be related to the ability of the material to modify the tooth surface to promote reinforcement through the deposition of various mineral ions. Nanoseal application is expected to be an effective method for the prevention of root surface caries. Additionally, a Nanoseal layer on the tooth surface might allow effective permeability of ions for remineralization.

\section{ACKNOWLEDGMENTS}

This study was supported by a Grant-in-Aid for Scientific Research No. 24659846 from the Japan Society for the Promotion of Science and Iwadare Scholarship Foundation.

\section{REFERENCES}

1) Griffin SO, Griffin PM, Swann JL, Zlobin N. Estimating rates of new root caries in older adults. J Dent Res 2004; 83: 634638.

2) Ettinger RL. Epidemiology of dental caries. A broad review. Dent Clin North Am 1999; 43: 679-694.

3) Locker D, Slade GD, Leake JL. Prevalence of and factors associated with root decay in older adults in Canada. J Dent Res 1989; 68: 768-772.

4) Saunders RH Jr, Meyerowitz C. Dental caries in older adults. Dent Clin North Am 2005; 49: 293-308.

5) Kassab MM, Cohen RE. The etiology and prevalence of gingival recession. J Am Dent Assoc 2003; 134: 220-225.

6) Imazato $\mathrm{S}$, Ikebe $\mathrm{K}$, Nokubi T, Ebisu S, Walls AWG. Prevalence of root caries in a selected population of older adults in Japan. J Oral Rehabil 2006; 33: 137-143.

7) Banting DW, Papas A, Clark DC, Proskin HM, Schultz M, Perry R. The effectiveness of $10 \%$ chlorhexidine varnish treatment on dental caries incidence in adults with dry mouth. Gerodontology 2000; 17: 67-76.

8) Keltjens H, Shaeken T, van der Hoeven H. Preventive aspects of root caries. Int Dent J 1993; 43: 143-148.

9) Ogaard B, Rølla G, Arends J. In vivo progress of enamel and root surface lesions under plaque as a function of time. Caries Res 1988; 22: 302-305.

10) Donovan T. Critical appraisal: Protocol for the prevention and management of root caries. J Esthet Restor Dent 2008; 20: 405-411.

11) Featherstone JDB. Fluoride, remineralization and root caries. Am J Dent 1994; 7: 271-274.

12) Bradshaw DJ, Marsh PD, Hodgson RJ, Visser JM. Effects of glucose and fluoride on competition and metabolism within in vitro dental bacterial communities and biofilms. Caries Res 2002; 36: 81-86.

13) Lynch E, Baysan A. Reversal of primary root caries using a dentifrice with a high fluoride content. Caries Res 2001; 35: 60-64.

14) Leake JL. Clinical decision making for caries management in root surfaces. J Dent Educ 2001; 65: 1147-1153.

15) Heijnsbroek M, Paraskevas S, Van der Weijden GA. Fluoride interventions for root caries: a review. Oral Health Prev Dent 2007; 2: 145-152.

16) Featherstone JD. The science and practice of caries prevention. J Am Dent Assoc 2000; 131: 887-899.

17) Han L, Okiji T. Effects of a novel fluoride-containing aluminocalciumsilicate-based tooth coating material (Nanoseal) on enamel and dentin. Am J Dent 2013; 26: 191195.

18) Han L, Ishizaki H, Fukushima M, Okaji T. Effect of a prototype fluoride-containing tooth surface coating material on enamel and dentin surfaces(in Japanese). Jpn J Conserv Dent 2012; 55: 53-59.

19) Ten Cate JM, Duijsters PP. Alternating demineralization and remineralization of artificial enamel lesions. Caries Res 1982; 16: 201-210.

20) Matsuda Y, Komatsu H, Murata Y, Tanaka T, Sano H. A newly designed automatic pH-cycling system to simulate daily $\mathrm{pH}$ fluctuations. Dent Mater J 2006; 25: 280-285.

21) Arends J, Ten Bosch JJ. Demineralization and remineralization evaluation techniques. J Dent Res 1992; 71: 924-928.

22) White DJ, Faller RV, Bowman WD. Demineralization and remineralization evaluation techniques -added considerations. J Dent Res 1992; 71: 929-933.

23) Paschos E, Kleinschrodt T, Clementino-Luedemann $T$, Huth KC, Hickel R, Kunzelmann KH, Rudzki-Janson I. Effect of different bonding agents on prevention of enamel demineralization around orthodontic brackets. Am J Orthod Dentofacial Orthop 2009; 135: 603-612.

24) Ryou H, Niu LN, Dai L, Pucci CR, Arola DD, Pashley DH, Tay FR. Effect of biomimetic remineralization on the dynamic nanomechanical properties of dentin hybrid layers. J Dent Res 2011; 90: 1122-1128.

25) Walter R, Duarte WR, Pereira PN, Swift EJ Jr, Heymann HO, Arnold RR. Effect of resin adhesive systems on root caries formation in vitro. Quintessence Int 2008; 39: 33-37.

26) Hamba H, Nikaido T, Inoue G, Sadr A, Tagami J. Effects of CPP-ACP with sodium fluoride on inhibition of bovine enamel demineralization: a quantitative assessment using microcomputed tomography. J Dent 2011; 39 :405-413.

27) Damen JJ, Exterkate RA, Ten Cate JM. Reproducibility of TMR for the determination of longitudinal mineral changes in dental hard tissues. Adv Dent Res 1997; 11: 415-419.

28) Zou W, Hunter N, Swain MV. Application of polychromatic $\mu \mathrm{CT}$ for mineral density determination. J Dent Res 2011; 90: 18-30.

29) Lo EC, Zhi QH, Itthagarun A. Comparing two quantitative methods for studying remineralization of artificial caries. J Dent 2010; 38: 352-359.

30) Neves Ade A, Coutinho E, Vivan Cardoso M, Jaecques SV, Van Meerbeek B. Micro-CT based quantitative evaluation of caries excavation. Dent Mater 2010; 26: 579-588.

31) Burghardt AJ, Kazakia GJ, Laib A, Majumdar S. Quantitative assessment of bone tissue mineralization with polychromatic micro-computed tomography. Calcif Tissue Int 2008; 83: 129138.

32) Greener EH, Harcourt JK, LAutenschlager EP. Materials science in dentistry. 1st ed. Baltimore: Waverly Press Inc; 1972. P. 244-249.

33) Saito T, Toyooka H, Ito S, Crenshaw MA. In vitro study of remineralization of dentin: effects of ions on mineral induction by decalcified dentin matrix. Caries Res 2003; 37: 445-449.

34) Moreno EC. Role of Ca-P-F in caries prevention: chemical aspects. Int Dent J 1993; 43: 71-80.

35) Chow LC. Tooth-bound fluoride and dental caries. J Dent Res 1990; 69: 595-600.

36) Orchardson R, Gillam DG. Managing dentin hypersensitivity. J Am Dent Assoc 2006; 137: 990-998. 